\title{
Understanding Redox Effects on Supported Bimetallic Particles
}

\begin{abstract}
C.E. Kliewer
ExxonMobil Corporate Strategic Research, 1545 Route 22 East, Annandale, NJ 08801

Supported bimetallic particles are frequently used in catalysis. During chemical reactions, these structures may become exposed to cyclic redox (reduction/oxidation) conditions. It is well-established that environmental changes can dramatically affect a metal particle's shape and/or size..$^{1-8}$ Thus, highly specialized reactors for ex-situ TEMbased studies have been developed to treat materials under tightly controlled process conditions and provide a more fundamental, science-based understanding of these nanostructural transformations. ${ }^{3,6,9,10}$ This study examines the effect of changing environmental conditions on the morphology of AuSn bimetallic particles and reveals the potential impact of redox cycling treatments on catalytic behavior.
\end{abstract}

An Edwards high vacuum evaporator was used to deposit AuSn particles onto a holey carbon-coated support film. The supported metal particles were thermally treated $(2 \mathrm{~h}$, $500{ }^{\circ} \mathrm{C}, 1 \mathrm{~atm} 99.999 \% \mathrm{H}_{2}$ ) in a reactor especially designed for ex-situ TEM studies. ${ }^{3.10}$ The material was transferred from the reactor into the TEM under inert conditions ${ }^{3,10}$ and then examined in the bright field (BF) imaging mode of a Philips CM200F operated at an accelerating voltage of $200 \mathrm{kV}$. Randomly-selected areas were imaged and "mapped out" for re-examination. Core (Au-rich) - shell (Sn-rich) particles of varying sizes were clearly evident after the initial reduction (Figure 1a). The material was then transferred back into the reactor, and an ISCO pumping system was used to create hydrothermal treatment conditions $\left(0.25 \mathrm{~h}, 100{ }^{\circ} \mathrm{C}, 1 \mathrm{~atm} 75: 25\right.$ steam/air). A subsequent re-examination of the "mapped" regions indicated that the core-shell type structures were still present but that the shell thicknesses had increased due to preferential oxidation of the Sn (Figure 1b).

The particles were given a final reduction treatment $\left(2 \mathrm{~h}, 500{ }^{\circ} \mathrm{C}\right.$, $\left.1 \mathrm{~atm} 99.999 \% \mathrm{H}_{2}\right)$ and then returned to the TEM following an inert transfer protocol. ${ }^{3,10}$ Re-examination of the previously "mapped" areas revealed core-shell structures (Figure 1c). However, some metal particle growth was evident. As the Sn shell thickness increased during hydrothermal oxidation, the distance between adjacent bimetallic particles decreased and certain particles began to "touch" each other. During the final reduction step, metal particles that were "touching" after the hydrothermal oxidation began to coalesce (circled areas in Figure 1) in an effort to minimize the system's overall surface energy. As metal particle size increases with time (via subsequent and/or more severe oxidation treatments), active surface area decreases. Hence, controlling the interparticle spacing 
in core-shell structures used under cyclic redox conditions can be an important variable in maintaining catalytic activity.

\section{References:}

1. C.E. Kliewer et. al. Proceedings Microscopy \& Microanalysis 6 (2000) 378.

2. C.E. Kliewer et. al. Proceedings Microscopy \& Microanalysis 7 (2001) 1214.

3. C.E. Kliewer et. al. Journal of Microscopy \& Microanalysis 12 (2006) 135.

4. C.E. Kliewer et. al. Proceedings Microscopy \& Microanalysis 8 (2002) 1408CD.

5. C.E. Kliewer et. al. Proceedings Microscopy \& Microanalysis 9 (2003) 920CD.

6. G. Kiss et. al. Journal of Catalysis 217 (2003) 217.

7. C.E. Kliewer et. al. Proceedings Microscopy \& Microanalysis 12 (2006) 816CD.

8. C.E. Kliewer et. al. Proceedings Microscopy \& Microanalysis 14 (2008) 1122CD.

9. L.F. Allard et. al. Proceedings Microscopy \& Microanalysis 3 (1997) 595.

10. C.E. Kliewer et. al. Proceedings Microscopy \& Microanalysis 1 (1999) 926.

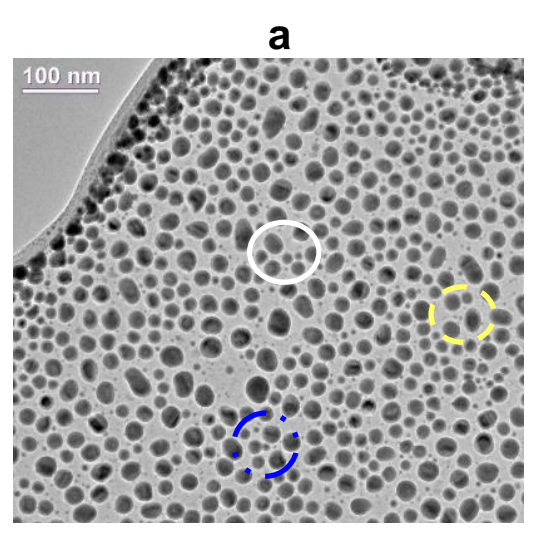

$\mathrm{H}_{2}, 500^{\circ} \mathrm{C}, 2 \mathrm{~h}$

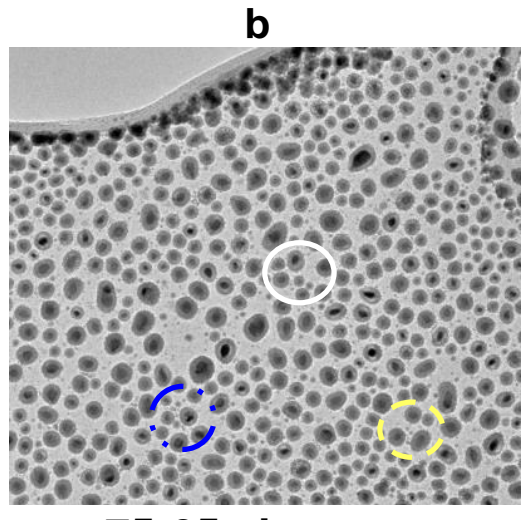

75:25 air:steam $100^{\circ} \mathrm{C}, 0.25 \mathrm{~h}$

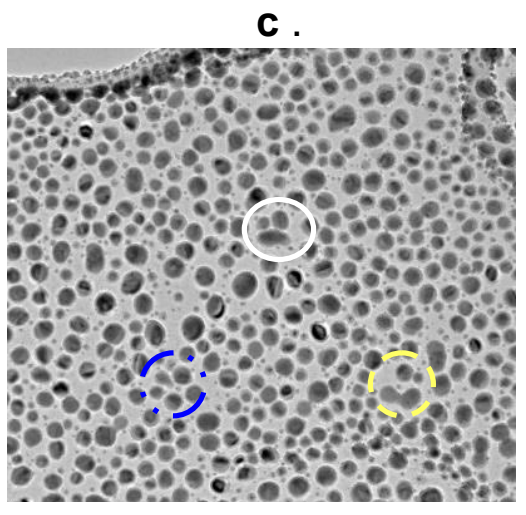

$\mathrm{H}_{2}, 500^{\circ} \mathrm{C}, 2 \mathrm{~h}$

Figure 1: Typical bright field (BF) TEM images reveal morphological changes in the same core $(A u)$ - shell $(S n)$ metal particles after the following thermal treatment sequence: (a) $2 \mathrm{~h}, 500{ }^{\circ} \mathrm{C}$ under $1 \mathrm{~atm} \mathrm{H}$, (b) $0.25 \mathrm{~h}, 100{ }^{\circ} \mathrm{C}$ under $1 \mathrm{~atm}$ 75:25 air:steam, and (c) $2 \mathrm{~h}, 500^{\circ} \mathrm{C}$ under $1 \mathrm{~atm} \mathrm{H}$. Circled regions identify the same metal particles in each image. 